\title{
Resistance to HSV-1 infection in the epithelium resides with the novel innate sensor, IFI-16
}

\author{
$\mathrm{CD}$ Conrady ${ }^{1}, \mathrm{M} \mathrm{Zheng}^{2}$, KA Fitzgerald ${ }^{3}, \mathrm{C}$ Liu $^{4}$ and DJJ Carr ${ }^{1,2}$
}

Toll-like receptors (TLRs) are innate sentinels required for clearance of bacterial and fungal infections of the cornea, but their role in viral immunity is currently unknown. We report that TLR signaling is expendable in herpes simplex virus (HSV)-1 containment as depicted by plaque assays of knockout mice (MyD88-/-, Trif $^{-/-}$and MyD88-/- Trif $^{-/-}$double knockout) resembling wild-type controls. To identify the key sentinel in viral recognition of the cornea, in vivo knockdown of the DNA sensor IFI-16/p204 in the corneal epithelium was performed and resulted in a loss of IFNregulatory factor-3 (IRF-3) nuclear translocation, interferon- $\alpha$ production, and viral containment. The sensor seems to have a similar function in other HSV clinically relevant sites such as the vaginal mucosa in which a loss of p204/IFI-16 results in significantly more HSV-2 shedding. Thus, we have identified an IRF-3-dependent, IRF-7- and TLR-independent innate sensor responsible for HSV containment at the site of acute infection.

\section{INTRODUCTION}

Herpes simplex virus type-1 (HSV-1) is a widespread, neurotropic, double-stranded DNA virus affecting $>60 \%$ of the world's population. ${ }^{1}$ The virus is an important clinical pathogen because of its ability to induce significant morbidity in the central nervous system and the cornea of both the immunocompetent and the immunosuppressed host. ${ }^{1,2}$ After mucocutaneous contact, HSV-1 initiates infection by first invading the host epithelium, replicating, and then gaining entry into sensory fibers where the virus is transported in a retrograde manner to neuronal cell bodies housed in the trigeminal ganglia. ${ }^{3}$ In most cases, the virus will then persist as a latent infection for the life of the host periodically reactivating to send infectious virions in an anterograde manner down various branches of the trigeminal nerve to erupt as "cold sores" on or near the labium. In rare but medically significant cases, HSV-1 is transported through the ophthalmic division of the trigeminal nerve to the immunologically privileged cornea where the virus initiates a sequence of inflammatory events that can eventually lead to corneal blindness because of significant immune-mediated scaring. ${ }^{2}$

During primary infection and subsequent HSV-1 reactivation in the cornea, in vitro studies suggest that innate cell membrane and cell compartment sensors (i.e., Toll-like receptors (TLRs)) are activated in response to specific viral invariant structures as displayed by responsiveness to TLR- 3 agonists. ${ }^{4,5}$ Once activated, TLRs in the cornea are believed to initiate signaling cascades through a myeloid differentiation primary response gene 88 (MyD88)- and/or TIR domain-containing adapter-inducing interferon (IFN)- $\beta$ (Trif) adaptor protein-dependent manner. The pathways then act to elicit nuclear factor- $\mathrm{\kappa B}$ and IFN-regulatory factor-3 (IRF-3) family activation to drive production of critical antiviral effector molecules such as double-stranded RNA-dependent protein kinase (PKR), RNase L, and Mx proteins by way of type 1 IFN signaling. ${ }^{5-9}$ In human corneas, TLR mRNA expression is upregulated during active herpetic stromal keratitis (HSK), ${ }^{10}$ and treatment of human corneal epithelial cell lines with polyinosinic-polycytidylic acid (poly (I:C)), a TLR-3 agonist, induces IFN- $\beta$ production. ${ }^{4}$ Furthermore, glucocorticoid treatment is believed to diminish TLR-3 signaling and subsequently enhance ocular susceptibility to viral infection. ${ }^{11}$ Conversely, others have reported a role for TLR signaling in initiating immunopathology in the cornea. ${ }^{12}$ Taken together, these results suggest a significant role for TLR signaling in both the containment of HSV-1 and the pathological outcomes of the cornea. However, none of the aforementioned studies implicating TLRs as the innate sensor of HSV-1 in the cornea incorporated in vivo models capable of identifying the innate sentinel initiating IFN production and the sensor's role in containing viral replication.

\footnotetext{
${ }^{1}$ Department of Microbiology, Immunology, University of Oklahoma Health Sciences Center, Oklahoma City, Oklahoma, USA. ²Department of Ophthalmology, University of Oklahoma Health Sciences Center, Oklahoma City, Oklahoma, USA. ${ }^{3}$ Division of Infectious Diseases and Immunology, University of Massachusetts Medical School, Worcester, Massachusetts, USA. ${ }^{4}$ Department of Orthopedic Surgery and Cell Biology, New York University School of Medicine, New York, New York, USA. Correspondence: DJJ Carr (dan-carr@ouhsc.edu) 

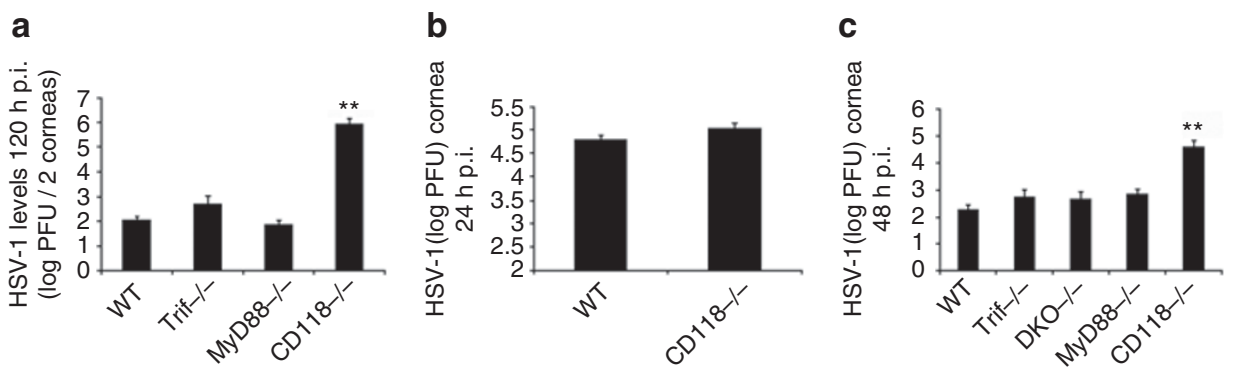

Figure 1 The loss of TLR signaling has not affect on viral containment in the cornea. WT, Trif ${ }^{-/-}, \mathrm{MyD}^{-/-}$, DKO, and CD118 $8^{-/-}$mice were infected with 1,000 p.f.u. HSV-1 per eye. At the indicated time pi, mice were killed and corneas harvested. Viral levels in the cornea were then determined by plaque assay at (a) 120 (b), 24, and (c), 48 h pi. Bars represent 2-4 experiments of 2-4 corneas per group per experiment and are expressed as the mean log p.f.u. per cornea \pm s.e.m. ${ }^{\star \star} P<0.01 ;{ }^{\star} P<0.05$ when comparing all groups. DKO, double knockout; HSV, herpes simplex virus; pi, post infection; TLR, Toll-like receptor; WT, wild type.

Thus, we set out to ascertain the in vivo role of TLR signaling during the innate immune response to HSV-1 in the cornea using mice deficient in TLR adaptor proteins $\left(\mathrm{MyD}^{-1-}\right.$, Trif $^{-1-}$, or both (double knockout)) to negate any TLR contribution to viral immunity. We hypothesized that type I IFN production required to prohibit viral replication was activated through signals emanating from TLRs and was the initial immune component necessary to control virus replication. Consequently, a loss of TLR signaling would result in a reduction in IFN production and an increase in HSV-1 susceptibility. Contrary to our hypothesis, we found that signals initiated by TLR recognition of HSV-1 were expendable, whereas the recently described nuclear-localized macrophage DNA sensor, p204/IFN-inducible protein 16 (IFI-16) ${ }^{13,14}$ mediated viral surveillance and innate immunity of the corneal epithelium crucial in the initial control of acute HSV-1 infection.

\section{RESULTS}

\section{Loss of TLR signaling has no effect on viral containment}

Previous work has shown a TLR-dependent signaling requirement in clearing bacterial and fungal infections of the cornea. ${ }^{15,16}$ Similarly, in vitro literature suggests that a loss of TLR pattern recognition, and a subsequent loss of activation of downstream signaling cascades disrupt HSV-1 containment in the cornea., ${ }^{41}$ To clarify the role of TLRs in the cornea in response to invading viruses, mice deficient in TLR adaptor proteins $\left(\mathrm{MyD} 88^{-/-}\right.$and Trif $^{-1-}$ ) were ocularly infected with HSV-1 and compared with wild-type (WT) controls and mice with an ablation of type 1 IFN signaling $\left(\mathrm{CD} 118^{-/-}\right)$rendering them highly susceptible to HSV-1 dissemination. ${ }^{17}$ Five days post infection (pi),

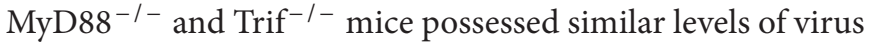
in the cornea as did WT controls, but significantly less infectious virus than did CD118 $8^{-/-}$mice (Figure 1a). To determine the earliest time point, a difference could be appreciated between $\mathrm{WT}$ and $\mathrm{CD} 118^{-/-}$mice; samples obtained at $24 \mathrm{~h}$ pi revealed no distinguishable differences between the two (Figure 1b). However, by $48 \mathrm{~h}$ pi, CD $118^{-/-}$mice harbored substantially more HSV-1 in the cornea than did WT controls (Figure 1c). Similar to 5 days pi, MyD88 $8^{-1-}$ and Trif $^{-1-}$ viral levels mirrored those of WT controls and were considerably lower than those of $\mathrm{CD} 118^{-/-}$mice at $48 \mathrm{~h}$ pi (Figure 1c). Higher inoculums of virus had similar outcomes (Supplementary Figure 1). To negate any TLR pathway redundancy, MyD88 $8^{-1-}$ and Trif $^{-1-}$ mice were backcrossed to produce double knockouts. Similar to WT, MyD88 ${ }^{-1-}$, and Trif ${ }^{-1-}$ mice, MyD88/Trif double knockout mice had equivalent levels of virus recovered in the cornea at $48 \mathrm{~h}$ pi (Figure 1c). Taken together, these results clearly demonstrated that TLR recognition was not required for resistance to HSV-1 infection of the cornea.

\section{IFN production despite a loss of TLR signaling}

As MyD88 $8^{-1-}$ and Trif $^{-1-}$ mice did not succumb to HSV-1 dissemination as seen in CD118 ${ }^{-/-}$mice (Supplementary Figure 1, data not shown), the results would suggest that other innate sensors were central to the activation of signaling pathways that result in type I IFN production and resistance to infection. We previously reported that IFN- $\alpha$ is secreted by infected resident cells and cells juxtaposed to infected cells of the corneal epithelium following HSV- 1 infection, whereas IFN- $\beta$ expression was restricted to cells residing in the limbus of the cornea. ${ }^{18}$ To investigate whether IFN- $\alpha$ production was altered or diminished in TLR adaptor-deficient mice, corneal whole mounts were evaluated by confocal microscopy. IFN- $\alpha$ production in Trif $^{-1-}$ and CD $118^{-/-}$mice was similar to WT controls and higher than areas devoid of HSV lesions in all mice (Figure 2a). IFN- $\alpha$ levels were reduced in the cornea of infected MyD88 ${ }^{-1-}$ mice, but still detectable relative to isotype controls and sites lacking HSV-1 (Figure 2a). This reiterated our previously published work in which infected cells (yellow arrows) and cells abutting infected cells (white arrows) were the primary source of IFN- $\alpha$ (Figure 2a). To further explore the consequences of a reduction in IFN- $\alpha$ expression in MyD88 $8^{-/-}$mice, we evaluated downstream IFN-inducible pathways including IFN-stimulatory gene-54 (ISG54) and oligosynthetase 1a (OAS1a) expression in the cornea following infection. In response to HSV-1 infection, the OAS1a expression was similar between $\mathrm{Trif}^{-1-}$ and WT mice at $24 \mathrm{~h}$ pi (Figure 2b). By comparison, the OAS1a expression was elevated in MyD88 ${ }^{-1-}$ mouse corneas compared with WT or Trif ${ }^{-1-}$ mouse samples at $24 \mathrm{~h}$ pi, although the levels did not reach significance. By $48 \mathrm{~h}$ pi, the OAS1a expression was no different between the three groups. ISG54 expression was not significantly different comparing WT with $\mathrm{MyD} 88^{-/-}$and 

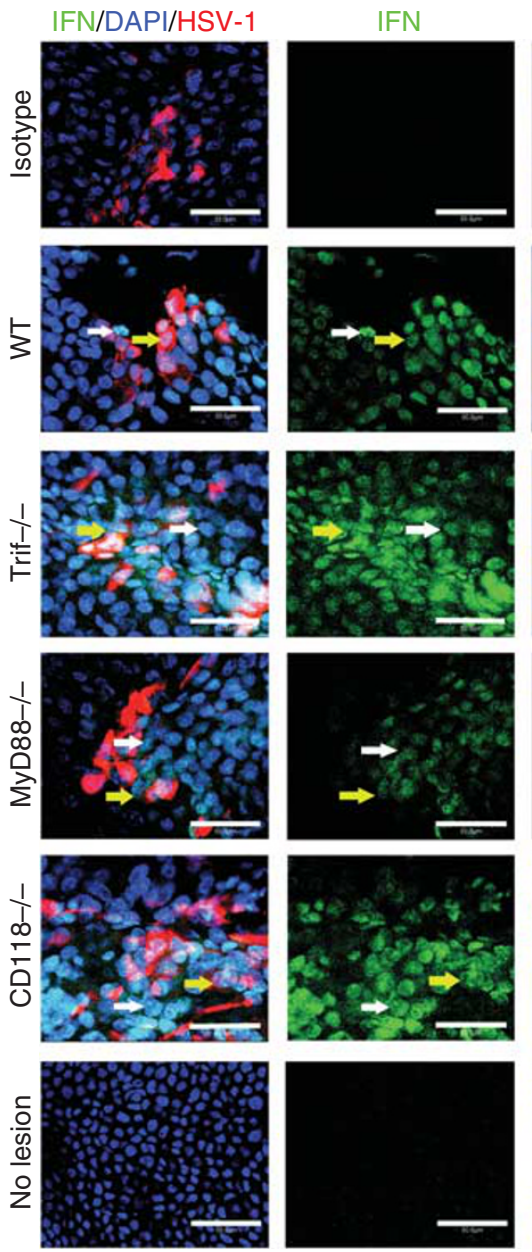

DAPI/HSV-1
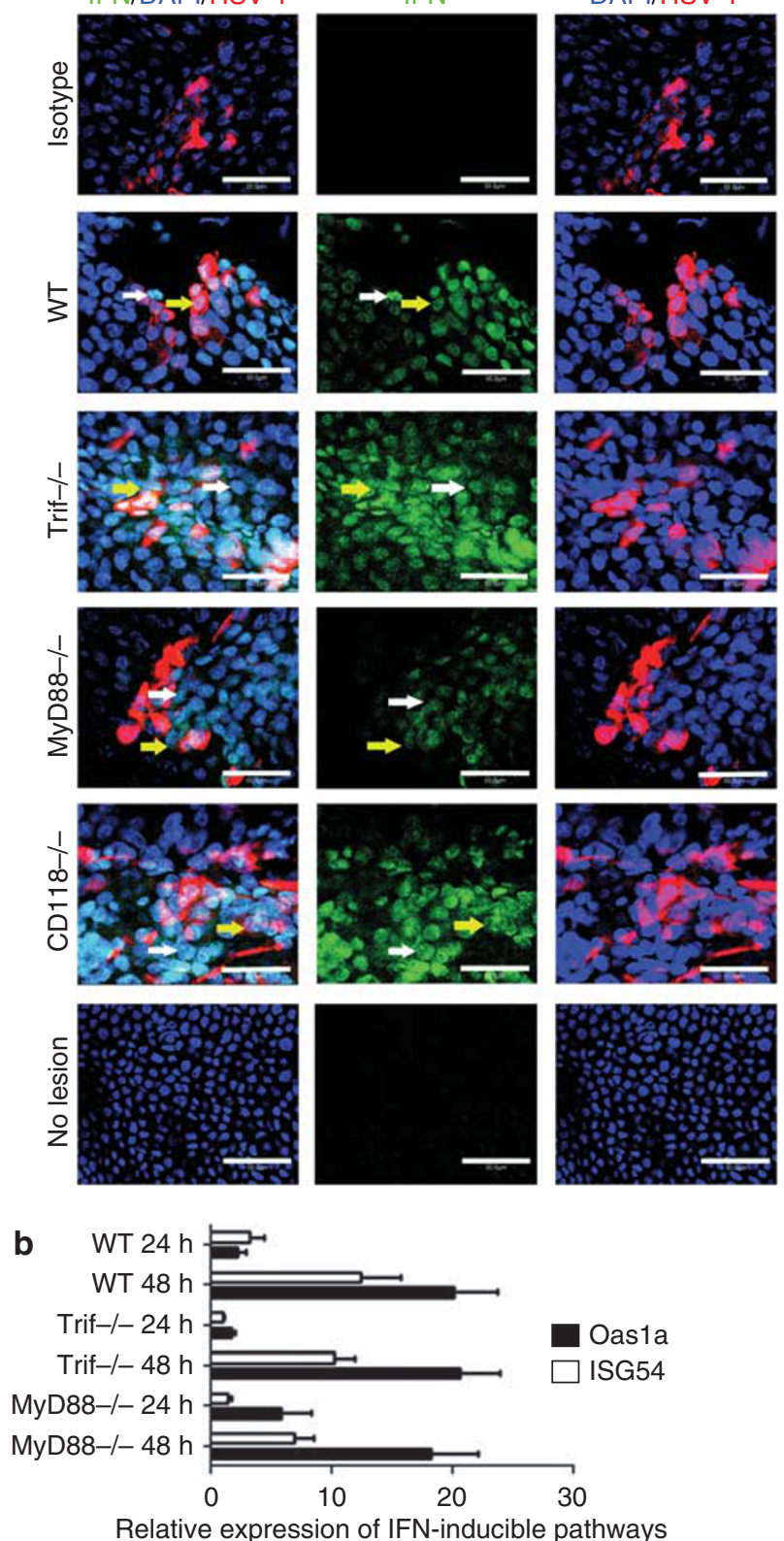

Figure 2 Unaltered IFN response despite a loss of TLR signaling. (a) WT, $\mathrm{Trif}^{-/-}, \mathrm{MyD}^{-/-}$, and CD118 $8^{-/-}$mice were infected with 1,000p.f.u. HSV-1 per eye. Forty-four to forty-eight hours pi, IFN production (green) was examined by confocal imaging relative to HSV-1 lesions (red) in the cornea ( $n=3-6$ corneas per group). Images are representative of two independent experiments acquired using a $\times 400$ objective with a digital zoom of $\times 10$. White arrows depict uninfected IFN- $\alpha$-expressing cells; yellow arrows depict HSV-infected IFN- $\alpha$-expressing cells; white bars, $50 \mu \mathrm{m}$. (b) Mice were infected with HSV-1 and 24 or $48 \mathrm{~h}$ pi, corneas were evaluated for mRNA expression of ISG54 and OAS1a by RT-PCR. Bars are normalized to uninfected controls and represent 2 independent experiments of 2-4 corneas per group plotted as relative expression \pm s.e.m. HSV, herpes simplex virus; IFN, interferon; ISG54, IFN-stimulatory gene-54; pi, post infection; OAS1a, oligosynthetase 1a; RT-PCR, reverse transcriptase-PCR; TLR, Toll-like receptor; WT, wild type.

Trif $^{-1-}$ mice at 24 and $48 \mathrm{~h}$ pi (Figure $2 \mathbf{b}$ ). As the downstream effector molecule of OAS activation, RNase L, contributes to viral surveillance in the cornea following HSV- 1 infection, ${ }^{6}$ the reduction in IFN- $\alpha$ expression observed in the cornea of MyD88 ${ }^{-1-}$ mice is not to a point that affects IFN-inducible, anti-viral pathways investigated. These data suggested an innate sensor other than TLRs as the principle sentinel in the cornea in response to HSV-1.

\section{p204/IFI-16 recognition of HSV-1 in the epithelium facilitates viral surveillance}

Although the loss of TLR signaling had minimal to no effect on viral containment and IFN- $\alpha$ production, the sensor that drives IFN- $\alpha$ production in the cornea during the acute HSV-1 infection remained elusive. To identify potential candidate sentinels in the cornea initiating IFN production, five innate sensors/adaptor proteins (DNA-dependent activator of IFNregulatory factors (DAI), mitochondrial antiviral signaling protein, absent in melanoma 2 , retinoic acid-inducible gene I (RIG-I), and IFN-inducible protein-16 (IFI-16/p204)) were evaluated by reverse transcriptase-PCR for upregulation following HSV-1 infection. DAI was the most highly induced sensor with a $>40$-fold increase following infection (Supplementary Figure 2). However, initial in vivo small-interfering RNA (siRNA) knockdown experiments of $\mathrm{DAI}^{19}$ had minimal to no effect on HSV-1 containment (Figure 3e, Supplementary Figure 2). The DNA sensor of macrophages, p204 (IFI16, the human homolog of mouse p204), next became the primary focus because of previous literary precedent depicting a role for the protein in initiating IFN production through an IRF-3dependent mechanism. ${ }^{13,14,20,21}$ To first establish the presence or absence of the mainly nuclear sensor, confocal microscopy and subsequent three-dimensional reconstructions of the cornea were used to localize p204 to the corneal epithelium with nuclear, perinuclear, and cytoplasmic staining (Figure 3a). To evaluate the role of p204 in innate recognition of HSV-1 in the cornea, initial analysis of the success of in vivo siRNA knockdown of the targeted protein was performed. The results showed a consistent reduction in global p204 protein expression $>50 \%$ of control-transfected and infected mice (Figures $3 \mathbf{b}$ and $\mathbf{4 a}$ ). Furthermore, cytoplasmic IRF-3 levels (Figure 3c) and IRF-3 nuclear translocation were severely diminished in WT mice treated with siRNA to p204 with corresponding HSV-1 titers significantly elevated compared with those of WT mice transfected with nonspecific siRNA at $48 \mathrm{~h}$ pi (Figure 3d and e). If normalized, the viral load difference between mice treated with nonspecific siRNA and siRNA to p204 were comparable to the differences seen between WT and CD118 ${ }^{-/-}$mice (Figure 1c). Furthermore, STING ${ }^{-1-}$ mice $^{22}$ contained significantly more virus in the cornea than did WT controls, yet had similar levels of virus as did CD118 $8^{-/-}$mice (Figure 3f). To confirm these results, antibody treatment, which has been shown to inhibit intracellular proteins, ${ }^{23}$ was used, and cytosolic p204 inhibition resulted in an increase in viral load (Figure 4b and c). p204 is known to translocate from the nucleus to the cytoplasm following viral infection, ${ }^{14}$ and the importance of this trafficking was further underscored by cytoplasmic antibody neutralization. To further substantiate a p204-dependent pathway finding in response to DNA viruses, in vivo (cornea) siRNA knockdown 
a

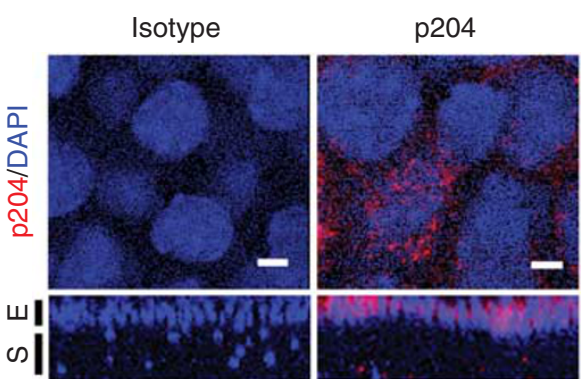

e

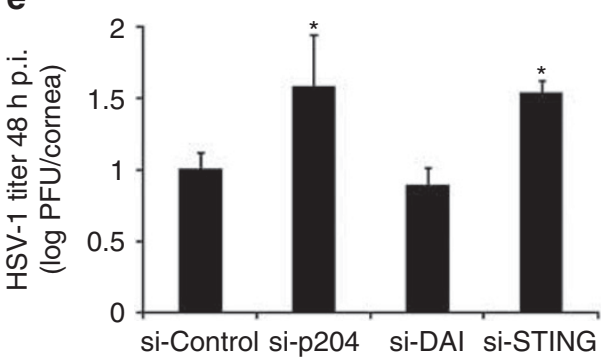

b

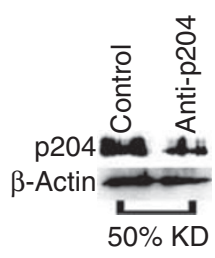

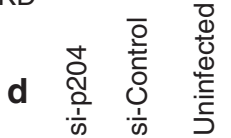

nIRF3

nTBP

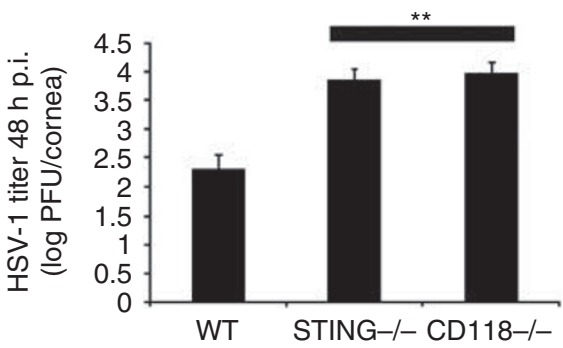

g
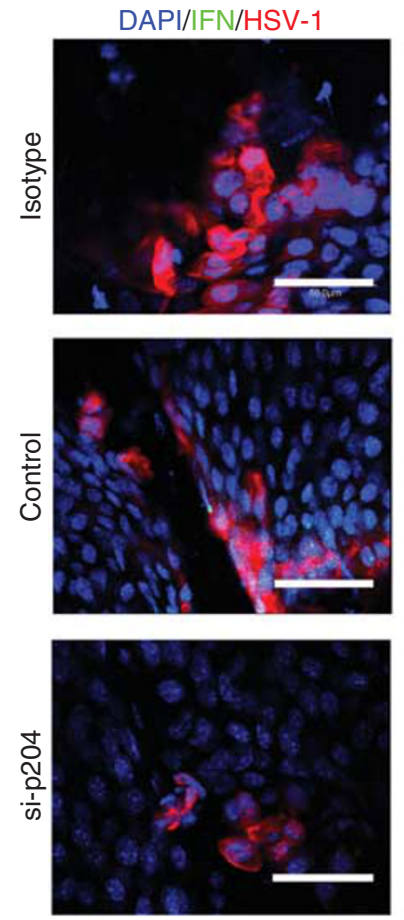
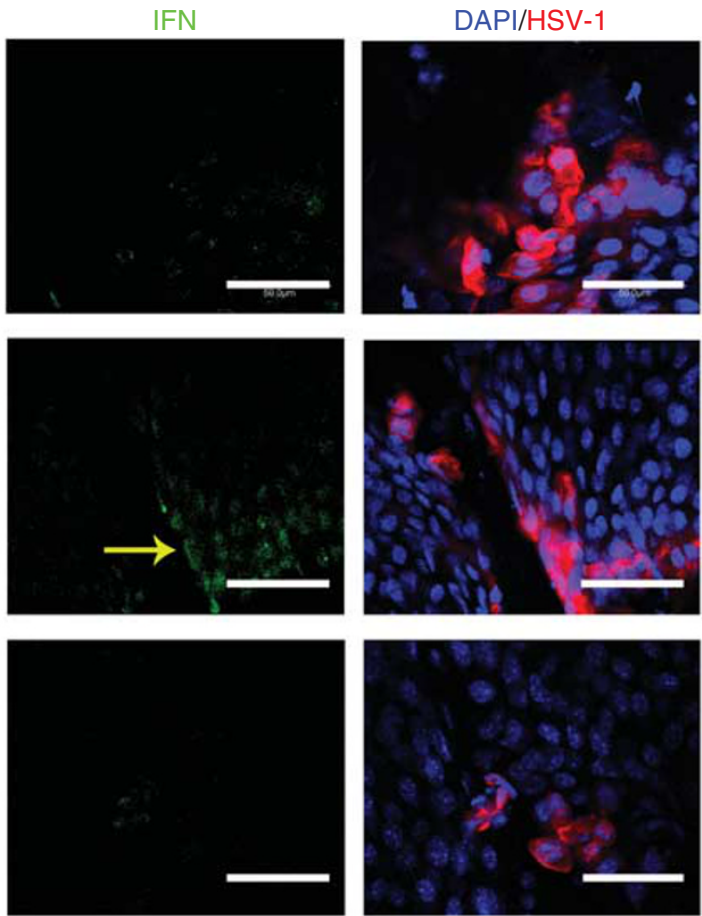

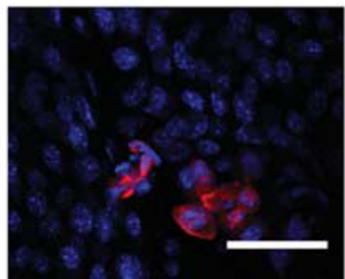

Figure 3 p204/IFI-16 expressed in the corneal epithelium drives resistance to HSV-1. (a) Uninfected WT mice were evaluated for p204 expression (red) by confocal microscopy and the sensor was found in the nuclear (blue), perinuclear, and cytoplasmic regions of the corneal epithelium using a $\times 400$ objective with a digital zoom of $\times 10$. The lower panels are 3D reconstructions of the cornea. S, stroma; E, epithelium; white bars, $50 \mu \mathrm{m} ; \mathrm{UI}$, uninfected. (b) In vivo siRNA knockdown of p204 was then performed in infected WT mice and consistently $>50 \%$ of levels of infected mice transfected with nonspecific siRNA. After siRNA knockdown of p204 and HSV-1 infection, IRF-3 cytoplasmic levels were diminished in siRNA-transfected groups (c); however, nuclear translocation of IRF-3 (d) was severely diminished 48h pi in the nuclear extract of WT corneas treated exclusively with si-p204 as compared with those of control siRNA-treated or uninfected mice. n, nuclear. (e) A loss of p204 or STING after siRNA knockdown resulted in significantly more infectious virus in the cornea compared with WT mice treated with nonspecific siRNA or siRNA to DAI ( $n=4-8$ corneas) as determined by plaque assay. ${ }^{\star} P<0.05$ comparing STING or p204 with DAI or control, values are representative of $2-3$ independent experiments. (f) WT, STING ${ }^{-/-}$, and CD $118^{-/-}$mice were infected with HSV-1 (1,000 p.f.u. per cornea) and viral load determined by plaque assay $48 \mathrm{~h}$ pi. Results represent 2 experiments of 2-4 corneas per group per experiment and are expressed as the mean log p.f.u. per cornea \pm s.e.m. ${ }^{\star \star} P<0.01$ compared with WT. (g) Trif ${ }^{-/-}$mice were transfected with nonspecific or anti-p204 siRNA and subsequently infected with HSV-1 (1,000 p.f.u. per cornea). Forty-eight hours pi IFN production (green) was analyzed by confocal microscopy in relation to herpetic lesions (red) in the cornea. White bars, $50 \mu \mathrm{m}$. DAl, DNA-dependent activator of IFNregulatory factor; HSV, herpes simplex virus; IFN, interferon; pi, post infection; siRNA, small-interfering RNA; 3D, three dimensional; WT, wild type. 
of STING (Supplementary Figure 2), the adaptor protein of p204, ${ }^{14}$ was evaluated for infectious content. The results show targeting STING expression resulted in a significant reduction in viral containment compared with controls and those of DAI knockdowns (Figure 3e). IFN production was then evaluated in transfected Trif $^{-1-}$ mice to negate siRNA activation of TLR-3 signaling and subsequent IFN production. ${ }^{24}$ Forty-eight hours pi, IFN production was impaired in mice treated with siRNA to p204 compared with mice transfected with nonspecific siRNA (Figure 3g). Taken together, these results suggested that p204 a

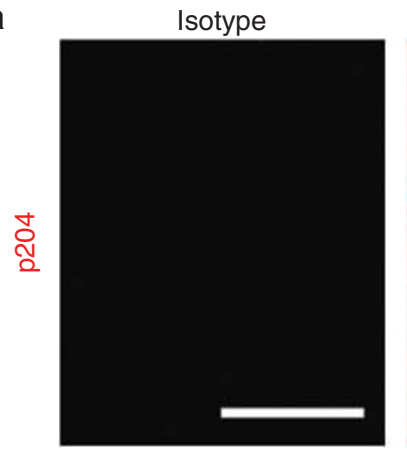

b

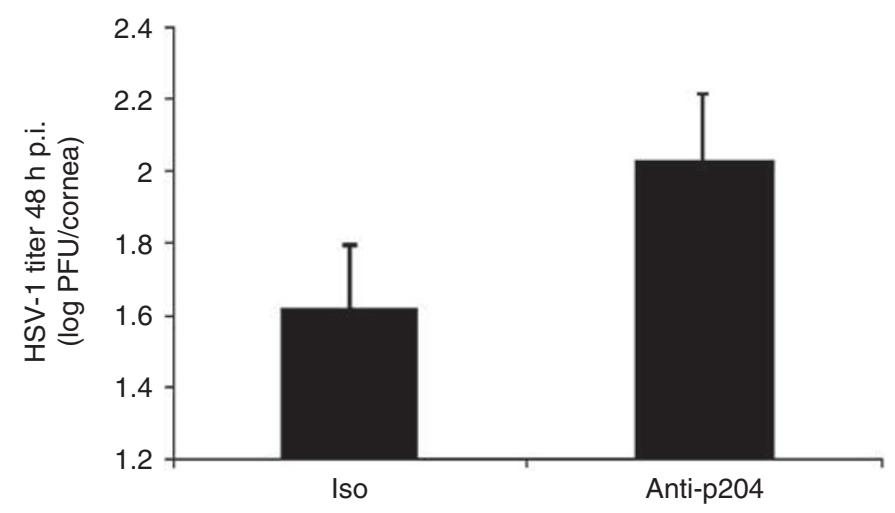

si-p204
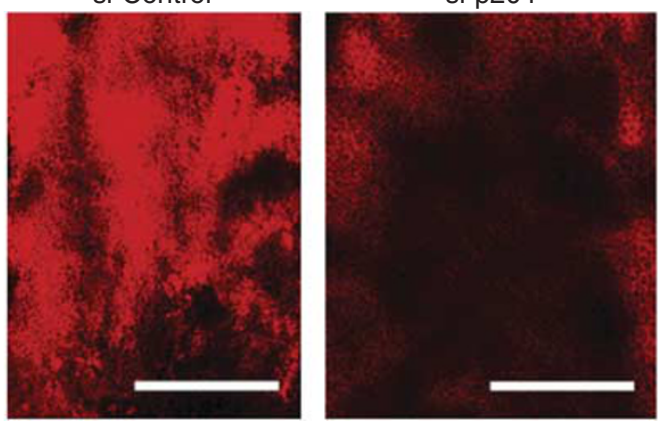

C
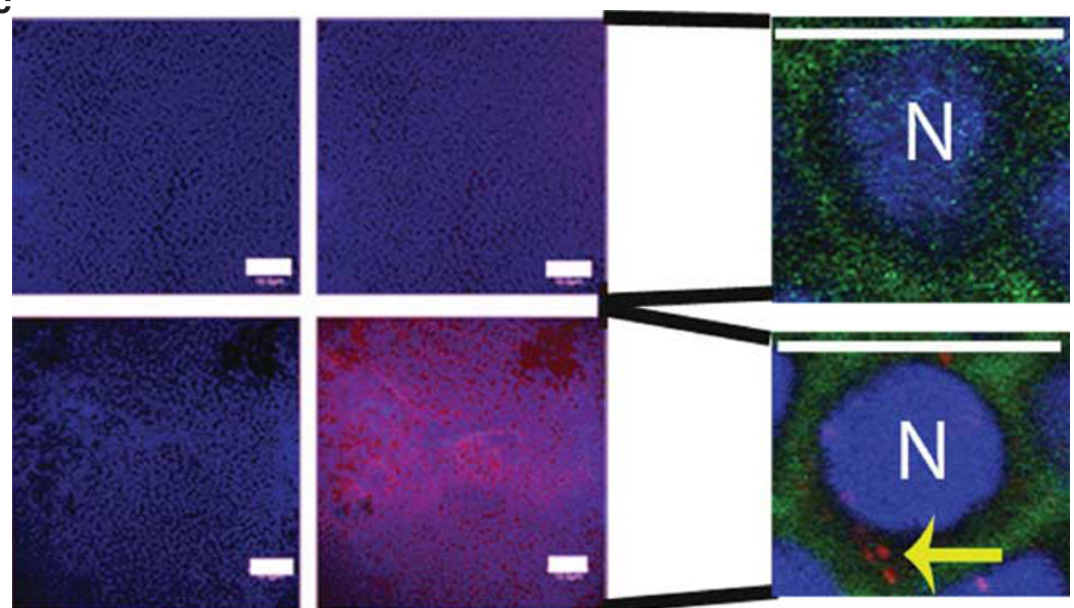

Figure 4 Cytoplasmic p204 neutralization results in an elevation in HSV-1 in the cornea. (a) To assess siRNA knockdown of p204, WT mice were transfected with either nonspecific siRNA or to p204. Twenty-four hours after transfection, knockdown was evaluated by confocal microscopy. White bar, $100 \mu \mathrm{m}$. (b) WT mice were infected with HSV-1 (1,000 p.f.u. per cornea) and subsequently administered a subconjunctival injection of isotype or anti-mouse p204 polyclonal antibody $(10 \mu \mathrm{g})$ to neutralize cytosolic protein. Forty-eight hour pi corneas were harvested and evaluated for viral titer by plaque assay. Bars represent two experiments of four corneas per group and are expressed as the mean log p.f.u. per cornea $\pm s . e . m$. (c, left panels) To confirm the ability of the p204 antibody (red) to access cellular cytoplasm, WT mice were infected with HSV-1 and administered a subconjunctival p204 injection (lower panel) or PBS (upper panel). The corneas were then fixed and stained with a DyLight549-conjugated anti-rabbit secondary and DAPI (blue, nuclei). Images represent two independent experiments of 2-4 corneas per group and were imaged at a magnification of $\times 200$. White bars, $100 \mu \mathrm{m}$. (panel c, right panels) To establish cellular location of the injected antibody, infected corneas were administered a subconjunctival injection of p204 antibody or PBS and were then stained with phalloidin (green, cell perimeters), p204 (red), and DAPI (blue) and imaged at ×600 with a digital zoom of 10 . White bar, $10 \mu \mathrm{m} ; \mathrm{N}$, nuclei, yellow arrows, cytoplasmic location of p204 antibody. HSV, herpes simplex virus; PBS, phosphate-buffered saline; siRNA, small-interfering RNA; WT, wild type. 


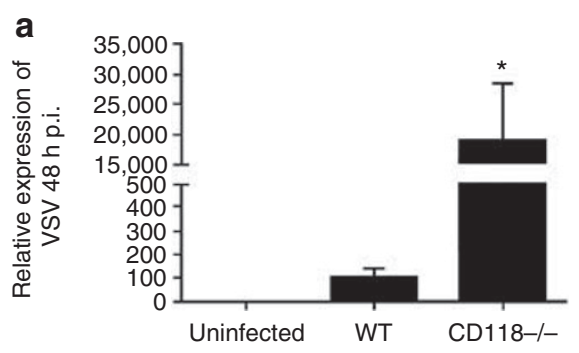

b

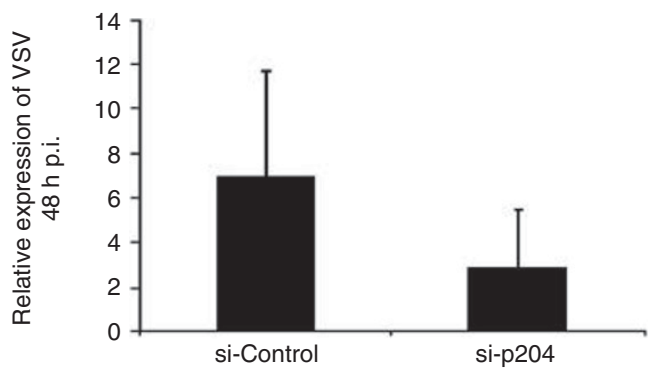

Figure 5 p204 drives innate resistance to HSV but not VSV in the cornea. (a) WT and CD $118^{-/-}$corneal sensitivity to VSV was compared $48 \mathrm{~h}$ pi following infection with 1,000 p.f.u. per eye VSV by RT-PCR. ${ }^{*} P<0.05$ comparing WT with CD118 ${ }^{-/-}$. (b) WT mice were transfected with either nonspecific siRNA or targeted to p204. Twenty-four hours after transfection, corneas were scarified and infected with VSV. Corneas were isolated $48 \mathrm{~h}$ pi and VSV levels were then evaluated by RT-PCR. Expression was normalized to $\beta$-actin levels. Bars represent 2 independent experiments of 2-4 corneas per group. HSV, herpes simplex virus; pi, post infection; RT-PCR, reverse transcriptase-PCR; siRNA, small-interfering RNA; WT, wild type; VSV, vesicular stomatitis virus.

was the initiator of HSV-1 immunity in the cornea of mice. To test the role of p204 in response to RNA viral infection, WT and $\mathrm{CD} 118^{-/-}$mice were infected with vesicular stomatitis virus. Forty-eight hours pi, CD $118^{-/-}$mice had significantly more vesicular stomatitis virus transcript expression than did WT, highlighting IFN production in RNA defense (Figure 5a). However, the loss of p204 did not lead to an increased susceptibility to the vesicular stomatitis virus (Figure $5 \mathbf{b}$ ). To ascertain whether the human equivalent of p204, IFI-16, resides in the human corneal epithelium as well, uninfected telomeraseimmortalized human corneal epithelial (THCE) cells were subjected to confocal microscopy and western blot analysis. The results clearly detected IFI-16 expression principally in the nucleus of THCE cells (Figure 6a) substantiated by the detection of a protein consistent with the apparent molecular weight of IFI16 of $90 \mathrm{kDa}$ (Figure $\mathbf{6 b}$ ). To firmly establish a direct interaction between IFI-16 and foreign DNA, IFI-16 pulldown of transfected biotinylated DNA in THCE cells was performed clearly showing binding of IFI-16 to foreign, guanine-cytosine (GC)-rich DNA, suggesting a role of the protein in cell immune surveillance of GC-rich viruses such as HSV-1 (Figure 6d). To substantiate the role of IFI-16, knockdown of IFI-16 in THCE cells (Figure 6e) resulted in a loss of viral containment not seen when RIG-I and TLR-9 knockdown was performed with similar efficiency (Figure 6c, data not shown). A loss of IFI-16 also resulted in a loss of nuclear translocation of IRF-3 (Figure 6f-h), but not nuclear translocation of IRF-7 in which nuclear protein levels are nearly indistinguishable (IFI-16 knockdown 98\% of control) (Figure 6i). It should also be noted that the mere infection of THCE cells with HSV-1 resulted in a loss of IFI-16 expression, suggesting a viral targeting of necessary host immune proteins (Figure 6e). To confirm that a loss of IFI-16 signaling reduced IFN signals, IFN- $\alpha$ driven CXCL10 expression ${ }^{25}$ was evaluated and a loss of IFI16 resulted in a loss of CXCL10 mRNA expression (Figure 6j). Therefore, the aforementioned results suggest a similar role of IFI-16 in humans as p204 in mice relative to HSV-1 surveillance within the cornea. However, the critical role of p204/IFI-16 is not restricted to the corneal epithelium, but the sensor likely initiates responses in the epithelium at other sites including the vaginal mucosa, which is another site commonly infected by HSV viruses. In particular, a reduction of p204 expression in WT mouse vaginal epithelium by siRNA (Figure 7c) results in a significant elevation in virus recovered from the vaginal lumen $48 \mathrm{~h}$ pi compared with siRNA control-treated or siRNA targeting DAI expression (Figure $7 \mathbf{a}$ and $\mathbf{b}$ ). To evaluate whether the sensor is also expressed in the epithelium of the skin, confocal microscopy was used to identify the presence of IFI-16 in human primary keratinocytes (Figure 7d). Although untested, this suggested that the sensor may have a role in HSV recognition of the skin.

\section{DISCUSSION}

In the study described herein, we have established to the best of our knowledge the first in vivo, TLR-independent innate immune response linked to containment of a clinically important viral pathogen in the cornea and genital epithelium. Despite a loss of TLR adaptor proteins and subsequent downstream TLR signaling, MyD88 $8^{-1-}$ and Trif $^{-/-}$mice showed competent IFN production in the cornea that resulted in viral surveillance equivalent to that of WT animals. In mice that are unresponsive to type I IFN (CD118 $\left.8^{-/-}\right)$, HSV-1 replicates in an uninhibited manner and rapidly disseminates throughout the host underscoring the critical role of IFNs in viral immunity. ${ }^{17,26}$

In tissues and cells found outside the cornea, HSV-1 surveillance is facilitated by TLRs, cytosolic RNA and DNA sensors, mitochondrial-associated proteins, and nuclear-localized sensors that act to drive IFN production necessary in viral containment. ${ }^{14,19,27}$ The presence of several of these sentinels in a single cell type highlights the considerable redundancy found in most cells. However, in certain tissues like the brain, TLR-3 seems to mediate a major portion of HSV-1 surveillance. Patients with TLR-3 deficiencies are more susceptible to herpes encephalitis than are healthy controls yet not other tested pathogens, ${ }^{28-30}$ emphasizing the importance of TLR-3 in the central nervous system in response to HSV-1. In the cornea, p204 facilitates the innate immune response to HSV-1 evident by increased susceptibility to virus replication when protein expression is compromised. In fact, if viral titers of siRNA-treated mice are normalized to unaltered WT levels $48 \mathrm{~h}$ pi, viral levels in mice treated with siRNA to p204 closely resemble those found in highly susceptible $\mathrm{CD} 118^{-1-}$ mice (5.4-4.99), whereas nonspecific siRNA treatment results in levels identical to WT (2.4-2.4). 
a

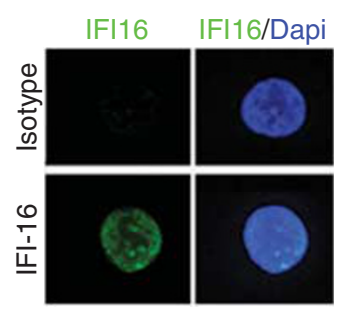

b

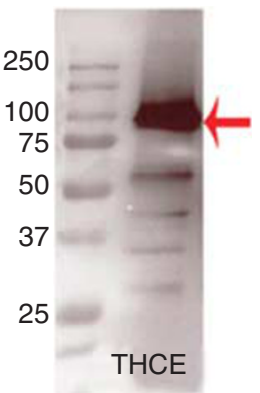

C

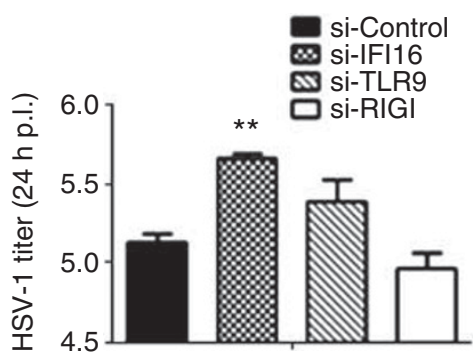

e

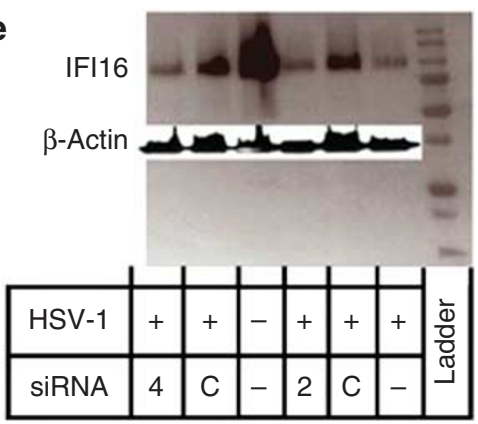

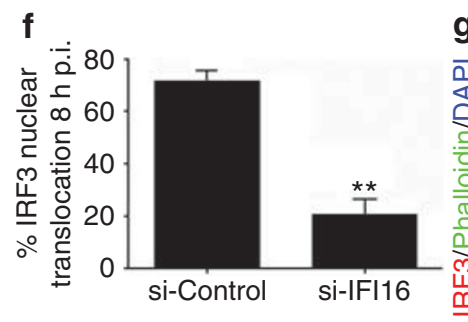
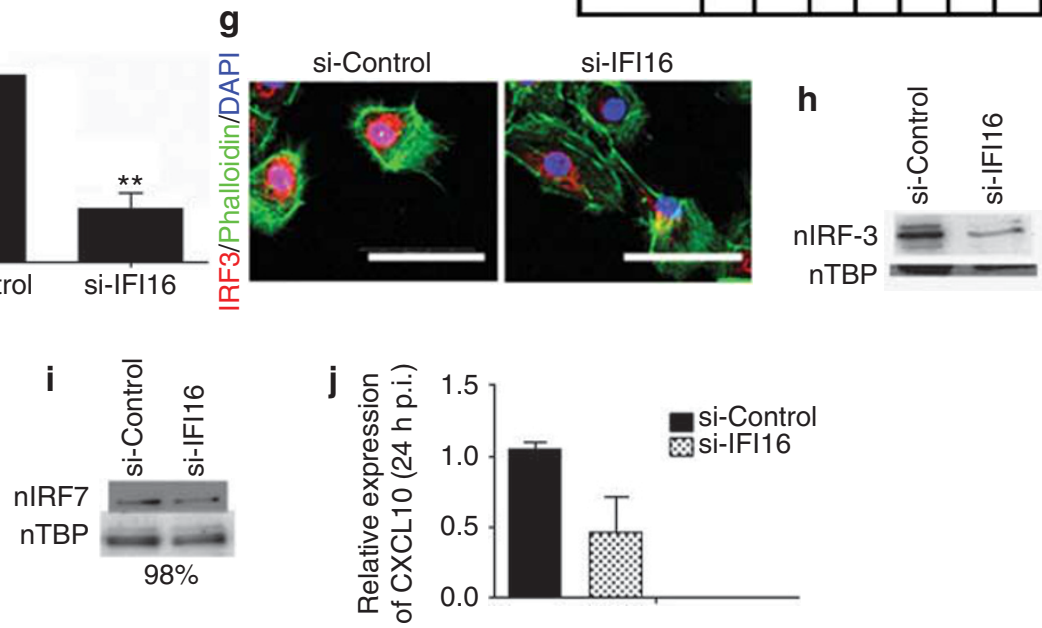

Figure 6 Human IFI-16 drives innate resistance in the cornea to HSV-1. (a) The human homolog, IFI-16 (green), was localized to the nucleus (blue) of uninfected THCE cells by confocal imaging using a $\times 400$ objective and digital zoom of $\times 10$. White bars, $200 \mu \mathrm{m}$. (b) Imaging was confirmed by western blot of uninfected THCE cells indicating the presence of the $\sim 90 \mathrm{kDa}$ protein as highlighted by the red arrow. (c) THCE cells were transfected with 2 pmol of control siRNA or siRNA to IFI-16, TLR-9, or RIG-I and infected $4 \mathrm{~h}$ later. Twenty-four hours pi, infectious content was evaluated by plaque assay ( $n=4-6$ per group, 2 independent experiments). ${ }^{* *} P<0.01$ comparing siRNA IFI-16 with siRNA control-treated groups. (d) To confirm a direct interaction between the DNA sensor (IFI-16) and HSV-1, ChIP pulldown of a biotinylated 50mer of DNA (single- or double-stranded, GC or non-GC rich) with IFI-16 antibody was performed in THCE cells and then subsequently identified by western blot analysis. (e) IFI-16 knockdown was performed in THCE cells with varying concentrations of control and anti-IFI-16 siRNA ( 2 or 4 pmol). Knockdown was confirmed by western blot. (f-h) After IFI-16 knockdown, IRF-3 nuclear translocation ( $\mathbf{g}$ ) was severely diminished $8 \mathrm{~h}$ pi as seen by confocal microscopy (quantified in panel f) and western blot analysis (panel h). n, nuclear; white bar, $50 \mu \mathrm{m}$. Images and blots are representative of 2-4 independent experiments. (i) However, IRF-7 nuclear translocation was no different (levels $98 \%$ of control) comparing IFI-16 knockdown with control-transfected THCE cells. Image represents two independent experiments. (j) THCE cells were transfected and infected. Twenty-four hours pi, CXCL10 expression was evaluated by RT-PCR. Results are representative of 2 independent experiments of 2-3 samples per group and expressed as the relative expression \pm s.e.m. ChIP, chromatin immunoprecipitation; HSV, herpes simplex virus; pi, post infection; RT-PCR, reverse transcriptase-PCR; siRNA, small-interfering RNA; THCE, telomerase-immortalized human corneal epithelial; TLR, Toll-like receptor.

This suggests that IFI-16/p204 is the principle initiator of IFN production in the cornea. Thus, although a new sensor, DDX41, has recently been shown to signal through a STING-dependent manner similar to that of $\mathrm{p} 204,{ }^{31}$ the loss of p204 seems to facilitate the major if not sole contribution to innate immunity of the cornea as seen by a loss of viral containment similar to that of both CD118 $8^{-/-}$and STING ${ }^{-/-}$mice. These results were confirmed by antibody inhibition of p204 resulting in an increase in infectious HSV-1 highlighting the importance of the cytoplasmic fraction of p204, which is a protein localized to the epithelium and some hematopoietic lineages and has been shown to inhibit ribosomal RNA transcription, to have a role in cell growth arrest, and most recently, to drive IFN production in response to intracellular HSV-1 DNA. ${ }^{13,14,32,33}$ Consequently, 

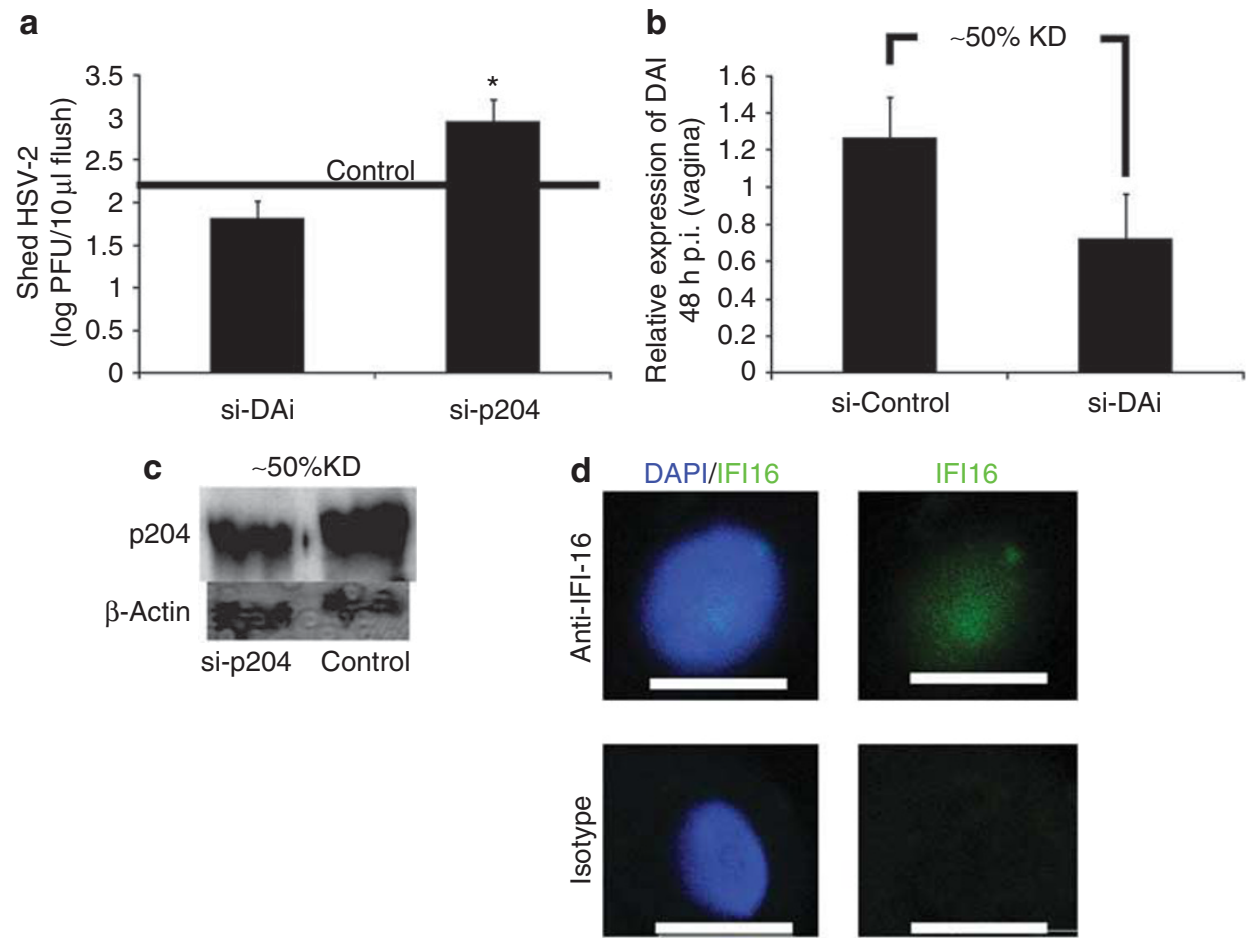

Figure 7 p204/IFI-16 drives epithelial resistance to HSV. To evaluate the role of p204/IFI-16 in other epithelial tissues, WT mice were transfected and infected with 2,000 p.f.u. per vagina. Viral shedding into the vaginal lumen (a) was evaluated by plaque assay $48 \mathrm{~h}$ pi. Results represent two independent experiments of two mice per group and are expressed as the mean log p.f.u. per tissue \pm s.e.m. ${ }^{*} P<0.05$ when comparing si-DAI with si-p204; black line represents levels seen in control-transfected mice. (b, c) RT-PCR or western blot analysis was used to confirm knockdown efficiency of DAI and p204 in the vaginal tissue respectively. (d) To identify the presence of IFI-16 (green) in human skin, adult primary keratinocytes were stained and imaged at $\times 200$ with a digital zoom of 2.5. Images represent 2 independent experiments of $1-2$ samples per group. White bars, $10 \mu \mathrm{m}$. DAl, DNA-dependent activator of IFN-regulatory factor; KD, knockdown; HSV, herpes simplex virus; pi, post infection; RT-PCR, reverse transcriptase-PCR; WT, wild type.

it seems plausible that a loss of this single protein, p204, and its downstream signaling events in the corneal epithelium, and in the epithelium in general, could explain the inability of $\mathrm{CD} 118^{-/-}$mice to mount an effective innate immune response. In particular, p204 binding of HSV DNA motifs recruits STING that acts to drive IFN production through an IRF-3-dependent, IRF-7-independent manner. ${ }^{14,34}$ IFNs then initiate JAK/STAT signaling to induce antiviral protein translation. ${ }^{35} \mathrm{~A}$ disruption in this cascade results in uncontrolled viral replication and spread into the central nervous system, ultimately resulting in encephalitis and death of the host.

Although innate redundancy seems widespread, it is not as extensive in some cells as others, possibly determined by embryological origins. TLRs have gained significant interest since their role in innate immunity was first established. ${ }^{36}$ However, they represent only one of several known viral sensors and may be expendable in certain tissues in response to specific pathogens as we report in the cornea in reference to HSV-1 infection. It may not be the major sensor, but p204 has a significant role in other epidermal tissues such as the stratified squamous epithelium of skin and vaginal mucosa where HSV replicates and elicits orolabial and genital lesions. This is highlighted by the observation in vaginal tissues that $\mathrm{CD} 118^{-/-}$ mice are extremely susceptible to HSV- $2,{ }^{37}$ and in mice lacking the p204 adaptor protein, STING, infection with HSV-1 results in more significant pathology and loss of IFN production. ${ }^{34}$
In relation to patients suffering from HSK, treatment with TLR antagonists may be of some clinical benefit. Although untested in our model, in vitro literature suggests that TLRs are functional ${ }^{4,10,11}$ and in vivo can initiate destructive inflammatory conditions. ${ }^{12,38,39}$ We hypothesize that inhibiting TLRs in the cornea could lead to better clinical outcomes in those suffering from herpes infections of the cornea due to a reduction in inflammation. ${ }^{12}$ In this study, we show that despite a loss of TLR signaling, initial control of HSV-1 replication remained sufficient. Thus, patients treated with TLR antagonists would likely have similar viral loads as untreated patients, yet less corneal inflammation if innate control of HSV-1 in humans mimics that of mice as it appears from our in vitro data. To further support our hypothesis, glucocorticoids are used clinically to effectively reduce inflammation in HSK and have been suggested to diminish TLR3 signaling in vitro. ${ }^{11} \mathrm{~A}$ reduction in TLR-3 signaling in the cornea may be one mechanism of action of broad-spectrum steroid treatments in reducing inflammation in HSK. Although TLR inactivation may be controversial, the need for more specific medications to alleviate corneal pathology due to HSK will continue to increase as do the number of immunocompromised patients who suffer from a higher incidence of herpes infections relative to the general public. ${ }^{40}$

In conclusion, we propose that $\mathrm{p} 204$ activates IFN- $\alpha$ production through a STING-dependent manner in response to HSV-1 
within resident cells of the cornea and likely other epithelial tissues. IFN- $\alpha$ drives the expression of antiviral pathways including PKR and RNase L, which work in concert to orchestrate an antiviral state in the cornea ${ }^{41}$ and to reduce the lytic replication of the virus proximal to the site of entry.

\section{METHODS}

Mice and virus. C57BL/6J WT and Trif-deficient $\left(\right.$ Ticam $^{-/-}$, Trif $^{-/-}$; C57BL/6J background) mice were purchased from Jackson Laboratory (Bar Harbor, ME) and housed in the Dean McGee Eye Institute's and Presbyterian Health Foundation's animal facilities alongside STINGdeficient (STING ${ }^{-/-}$), ${ }^{22} \mathrm{MyD}^{-1-} 8^{-12}$ and CD $118^{-/-}$mice $^{43}$ Trif $^{-/-}$ and $\mathrm{MyD} 88^{-/-}$mice were mated to generate double knockouts. Animal treatment was consistent with the National Institutes of Health Guidelines on the Care and Use of Laboratory Animals, whereas all experimental procedures were approved by the University of Oklahoma Health Sciences Center and Dean A. McGee Eye Institutes' Institutional Animal and Care Use Committees. HSV-1 McKrae and a clinical isolate of HSV-2 were passed as described previously. ${ }^{17,37}$ Vesicular stomatitis virus (Indiana strain) was originally obtained from Dr Robert Fleischmann (University of Minnesota).

In vivo HSV-1, HSV-2, and vesicular stomatitis virus infection. Male and female mice, aged 6-10 weeks, were anesthetized by an intraperitoneal injection of xylazine $(6.6 \mathrm{mg} \mathrm{kg})$ and ketamine $(100 \mathrm{mg} \mathrm{kg})$. Corneas were scarified with a $25-\mathrm{G} 1 \frac{112}{2}$ needle and tear film blotted. HSV-1 McKrae or vesicular stomatitis virus were resuspended $(1,000$ p.f.u. per eye) in $3 \mu$ l of sterile $1 \times$ phosphate-buffered saline (PBS) and topically applied onto each cornea. Female mice were vaginally infected with 2,000 p.f.u. per vagina resuspended in $20 \mu \mathrm{l}$ of $1 \times$ PBS 5 days after Depo-Provera (Pfizer, New York, NY) treatment.

Plaque assays. The corneas and $10 \mu \mathrm{l}$ vaginal washes of infected mice were harvested at designated times, resuspended in RPMI 1640 media containing $10 \%$ fetal bovine serum and antibiotic/antimycotic reagents (Invitrogen, Grand Island, NY) (referred to as normal media), and subjected to homogenization with a tissue miser for approximately 20-30 s as described previously. ${ }^{17}$ The supernatant was then clarified with a $10,000 \times g$ spin for $1.5 \mathrm{~min}$ and then serially diluted onto a confluent lawn of HSV-1 susceptible green monkey kidney (Vero) cells. Plaques were allowed to develop for $24-36 \mathrm{~h}$ and then counted using an inverted Zeiss microscope (Zeiss, Thornwood, NY).

Chromatin immunoprecipitation assay. In brief, THCE cells were transfected (as seen elsewhere) with 4 pmol of biotinylated (on $5^{\prime}$ end) single- or double-stranded GC-rich or $\triangle \mathrm{GC}$ DNA 50mers. Twenty-four hours after transfection, cells were fixed with paraformaldehyde, subjected to glycine, and washed three times. Cells were then lysed in SDS lysis buffer with protease inhibitors. Samples were then incubated with IFI-16 antibody (Santa Cruz, Santa Cruz, CA) for $2 \mathrm{~h}$ at $4^{\circ} \mathrm{C}$, except those samples used as controls. Samples were then incubated with protein A magnetic beads for $1 \mathrm{~h}$ at $4^{\circ} \mathrm{C}$ and then passed over a magnetic column. Samples were rinsed with several different washes as per the manufacturer's instructions (Miltenyi Biotec, Cambridge, MA). After elution, samples were run on an SDS gel and the biotin was probed for using strepavidin conjugated to horseradish peroxidase. Sequences for DNA 50mers are as follows:

\begin{tabular}{ll}
$\begin{array}{l}\text { DNA } \\
\text { 50mers }\end{array}$ & \\
\hline GC rich & 5'-ATGGCTCCGCCCCGCCGGGACCCCGCCCCC \\
& GGCCCGCCCCGGGCT-3' \\
$\Delta$ GC & 5'-ATGGCTCTGTGTTGCCGGGACCTGTGTTCCGG \\
& CCTGTGTTGGGCT-3' \\
\hline
\end{tabular}

Confocal imaging. WT, $\mathrm{Trif}^{-/-}, \mathrm{MyD} 88^{-/-}$, and $\mathrm{CD} 118^{-/-}$corneas were harvested at the indicated time pi. The corneas were fixed for $30 \mathrm{~min}$ with $4 \%$ paraformaldehyde at $4^{\circ} \mathrm{C}$ and subsequently washed three times with 1\% Triton X-100 (Bio-Rad, Hercules, CA) in PBS (PBS-Triton). Samples were then blocked overnight with either donkey serum (Jackson Immunology, West Grove, PA) or Fc block (BD Pharmingen, San Diego, $\mathrm{CA})$ at $4^{\circ} \mathrm{C}$ and washed with PBS-Triton. After the final wash, purified rabbit polyclonal antibody to $204^{13}$ or HSV-1 (Dakocytomation, Carpinteria, CA) was incubated overnight at $4^{\circ} \mathrm{C}$. The corneas were washed again and secondary antibodies conjugated to DyLite549 fluorochrome (Jackson Immunology) and fluorescein isothiocyanate-conjugated isotype, anti-IFN- $\alpha$, or anti-IFN- $\beta$ (PBL Biomedical, Piscataway, NJ) antibodies were incubated overnight at $4^{\circ} \mathrm{C}$. After three washes, DAPI (Vector Labs, Burlingame, CA) staining of nuclei was then performed overnight. Corneas were then mounted and imaged using an Olympus IX81-FV500 epiflourescent/confocal laser-scanning microscope (Olympus, Center Valley, PA). Images were analyzed with Fluoview software (Olympus). Three-dimensional reconstruction was performed by successive z-stack compilation using Fluoview software. THCE cells (a gift from Dr Jerry Shay, UT Southwestern) ${ }^{44}$ and adult primary keratinocytes (PromoCell, Heidelberg, Germany) were fixed and imaged in the same manner, except anti-IFI-16 (human p204 homolog) was purchased from Santa Cruz (clone 1G7) and human serum (Jackson Immunology) was used as the isotype control. Anti-IRF-3 polyclonal antibody recognizes both phosphorylated and unphosphorylated proteins (Abcam, Cambridge, MA). Phalloidin dye (Invitrogen) labels F-actin. To quantify IRF-3 nuclear translocation, images were captured, and all cells were counted in the visual field for IRF-3 translocation (co-localization with DAPI stain in the nucleus). The number of cells counted was then compared with the total number of cells in each visual field to calculate the percentage nuclear translocation.

In vitro transfection and infection. THCE cells were grown in 6-well plates to $50-70 \%$ confluency in keratinocyte serum-free medium containing bovine pituitary extract and epithelial growth factor (KSFM) at which point transfected with 2 or 4 pmol of either nonspecific or siRNA specific to IFI-16, TLR-9, or RIG-I (Invitrogen) in KFSM. Sequences are included below. After a 4-h incubation, KFSM was removed and replaced with fresh KFSM. Twenty-four hours after transfection, cells were infected with an multiplicity of infection of 0.001 of HSV-1 (strain McKrae). KFSM was then replaced after $1 \mathrm{~h}$ at $37^{\circ} \mathrm{C}$ with fresh KFSM, and cells and supernatant harvested $24 \mathrm{~h}$ pi. Cells were then used to confirm knock down by western blot of IFI-16 and viral titer to evaluate the role of IFI-16 in the human corneal epithelium in response to HSV-1 infection.

\begin{tabular}{ll}
\hline siRNA & \\
\hline si-Control & 5'-UUCUCCGAACGUGUCACGUTT-3' \\
si-hIFI16 & 5'-GGUGCUGAACGCAACAGAAUCAUUU-3' \\
si-p204 & 5'-CGGAGAGGAAUAAAUUCAUT-3' \\
si-STING & 5'-AACUGUUUCCGUCUGUGGGUUCUUG-3' \\
si-DAI & 5'-ACAGUCCAGACAGUCCACAUCAAAU-3' \\
si-hTLR9 & 5'-CUGGAAGAGCUAAACCUGA-3' \\
si-hRIGI & 5'-CAAGAAGAGUACCACUUAA-3' \\
\hline
\end{tabular}

DAI, DNA-dependent activator of IFN-regulatory factor; siRNA, smallinterfering RNA.

p204 antibody inhibition. WT mice were infected with 1,000 p.f.u. per eye. Thirty minutes pi, mice were administered a subconjunctival injection of $10 \mu \mathrm{g}$ of rabbit isotype or anti-p204 antibody (rabbit polyclonal). Forty-eight hours pi, the corneas were harvested and evaluated for viral content by plaque assay. For antibody detection, mice were subjected to the treatment described above, and corneas were harvested, fixed, and blocked as described previously. The corneas were then imaged by confocal microscopy. 
In vivo transfection. Five microliters of lipofectamine (Invitrogen) was mixed with $2 \mu \mathrm{l}$ of sterile Dulbecco's modified Eagle's medium lacking serum and antibiotics, whereas $1 \mu \mathrm{l}$ of control (negative control siRNA), anti-p204 siRNA, anti-DAI, or anti-STING (1 pmol; Stealth RNAi siRNA, Invitrogen) was mixed with $2 \mu$ l of Dulbecco's modified Eagle's medium. The solutions were then mixed after a $5 \mathrm{~min}$ incubation and topically applied to anesthetized mice whose corneas had been scarified with a $25-G 1 \frac{1}{2}$ " needle. Twenty-four hours after transfection, mice were infected with HSV-1 and evaluated for knockdown (consistently $>50 \%$ of control-transfected and infected WT mice) by western blot using a Kodak Imager (Kodak, Rochester, NY) or reverse transcriptase-PCR, viral titer, or IFN production. Vaginal mucosa was transfected in much the same manner, but volume doubled. In brief, 5 days after treatment with Depo-Provera, ${ }^{37}$ female mouse vaginal tracts were swabbed, and $2 \mu$ of nonspecific or specific si-RNA (si-DAI or si-p204) was mixed with $10 \mu \mathrm{l}$ of lipofectamine resuspended in $8 \mu \mathrm{l}$ serum-free Dulbecco's modified Eagle's medium. This was then applied intravaginally. Twenty-four after transfection, mice were infected with 2,000 p.f.u. per vagina HSV-2 (clinical isolate, Charity Hospital, New Orleans, LA).

IRF-3/7 nuclear translocation. Corneas were harvested $48 \mathrm{~h}$ pi from infected and uninfected mice and sonicated in cell lysis buffer with protease inhibitor (Calbiochem, Darmstadt, Germany) resuspended in T-PER (Thermo Scientific, Logan, UT) for 20 s. Samples were then rotated for $1 \mathrm{~h}$ at $4^{\circ} \mathrm{C}$ and subsequently centrifuged at $10,000 \times g$ for $15 \mathrm{~min}$. The supernatant was removed for analysis, whereas cell lysis buffer (Santa Cruz) was added to the pellet. Sample buffer (Bio-Rad) was then added and the mixture was boiled for $5 \mathrm{~min}$. Samples were then analyzed for protein content and $\beta$-actin and/or TATA-binding protein were used as the loading controls. Nuclear translocation of IRF-3 and IRF-7 was evaluated in THCE cells $8 \mathrm{~h}$ pi as described for mouse corneas.

Reverse transcriptase-PCR. Total RNA was isolated from WT (unaltered or transfected), MyD88 $8^{-/-}$, Trif $^{-1-}$, and CD $118^{-/-}$corneas and vaginal tissue at the indicated time pi using Trizol as per the manufacturer's guidelines (Invitrogen). cDNA was synthesized using avian myeloblastosis reverse transcriptase (Promega, Madison, WI) with oligo (dT) primers (Promega) and quantitative real-time PCR was performed using an iCycler (Bio-Rad) as described previously. ${ }^{45}$ Primer sequences can be found below or as described previously. ${ }^{46-48}$

\begin{tabular}{|c|c|}
\hline$\beta$-ActinF & 5'-CTTCTACAATGAGCTGCGTGTG-3' \\
\hline$\beta$-ActinR & 5'-TTGAAGGTCTCAAACATGATCTGG-3' \\
\hline STINGF & 5'-CCTAGCCTCGCACGAACTTG-3' \\
\hline STINGR & 5'-CGCACAGCCTTCCAGTAGC-3' \\
\hline DAIF & 5'-GGAAGATCTACCACTCACGTC-3' \\
\hline DAIR & 5'-CCTTGTTGGCAGATCATGTTG-3' \\
\hline AIM2F & 5'-TGGAACAATTGTGAATGGGCT-3' \\
\hline AIM2R & 5'-CACCAACACCTCCATTGT-3' \\
\hline RIGIF & 5'-CCACAAACTTGGAGAGTCACG-3' \\
\hline RIGIR & 5'-GAGTTTACAGATATGTTGCATG-3' \\
\hline MAVSF & 5'-CAAGAGCAAGAACCAGAACTG-3' \\
\hline MAVSR & 5'-GTGGAAGGGGCTGGAAGG-3' \\
\hline p204F & 5'-TCAGTTTCAGTAGCCACGGTAGCA-3' \\
\hline p204R & 5'-TGGTCCCAAACAAGTGATGGTGC-3' \\
\hline IFI16F & 5'-CCGTTCATGACCACGAGCATAGG-3' \\
\hline IFI16R & 5'TCAGTCTTGGTTTCAACGTGGT-3' \\
\hline VSVF & 5'-AATGACGATGAGACGATGCAATC-3' \\
\hline VSVR & 5'-CAAGTCACGCGTGACCATCT-3' \\
\hline
\end{tabular}

\section{Oas1aF 5'-CTTTGATGTCCTGGGTCATGT-3' \\ Oas1aR 5'-GCTCCGTGAAGCAGGTAGAG-3' \\ ISG54F 5'-TCTGATTCTGAGGCCTTGCA-3' \\ ISG54R 5'-TGCTGACCTCCTCCATTCTC-3'}

Western blots. Corneas and vaginal tissues were isolated from infected and uninfected mice. Samples were then resuspended in radio-immunoprecipitation buffer containing protease inhibitor (Santa Cruz) and subjected to a brief homogenization using a tissue miser. Samples were then allowed to solubilize for $20 \mathrm{~min}$ at $4^{\circ} \mathrm{C}$ and centrifuged for 15-20 min. Supernatant was evaluated for protein content (Bio-Rad). After electrophoresis at $99 \mathrm{~V}$, protein samples were transferred to polyvinylidene fluoride membranes soaked in methanol for $1 \mathrm{~h}$ at $99 \mathrm{~V}$. Membranes were washed in Tris buffer ( $\mathrm{pH} 7.6)$ and primary antibody (IRF-3 and IRF-7, Abcam) (1:1,000 dilution) incubated overnight at $4^{\circ} \mathrm{C}$. After three washes in Tris buffer ( $\left.\mathrm{pH} 7.6\right)$, samples were treated with secondary antibody (1:5,000 dilution) for $1 \mathrm{~h}$ at room temperature and washed again three times. Signal intensity was then evaluated (Pierce, Rockford, IL) on a Kodak imager.

Statistical analysis. Statistical analysis was conducted using a GBSTAT one-way analysis of variance followed by an ad hoc Tukey's $t$-test in experiments with more than two groups, whereas Student's $t$-test or two-way analysis of variance (THCE IRF-3 translocation only) was used to evaluate significance between only two variables. Significance was defined as a $P$-value $<0.05$ throughout the paper.

SUPPLEMENTARY MATERIAL is linked to the online version of the paper at http://www.nature.com/mi

\section{ACKNOWLEDGMENTS}

We thank Sara Moore, Linh Sramek, and Gabby Nguyen for technical support and Drs Helen Rosenberg, Shizuro Akira, and Russell E Vance for $\mathrm{CD}_{118^{-/-}, \mathrm{MyD}^{-/-} \text {, and STING }}^{-/-}$, mice respectively. We also thank Todd Wuest for reagents. Principal support for the study was from NIH Al053108 to DJJC. CDC was supported in part by NIAID training grant Al007633. Additional support includes an OUHSC Presbyterian Health Foundation Presidential Professorship award to DJJC, NIH/NCRR P20 RR017703, and an unrestricted grant from Research to Prevent Blindness.

\section{DISCLOSURE}

The authors declared no conflict of interest.

(C) 2012 Society for Mucosal Immunology

\section{REFERENCES}

1. Conrady, C.D., Drevets, D.A. \& Carr, D.J. Herpes simplex type I (HSV-1) infection of the nervous system: is an immune response a good thing? J. Neuroimmunol. 220, 1-9 (2010).

2. Duan, R., de Vries, R.D., Osterhaus, A.D., Remeijer, L. \& Verjans, G.M. Acyclovir-resistant corneal HSV-1 isolates from patients with herpetic keratitis. J. Infect. Dis. 198, 659-663 (2008).

3. Khanna, K.M., Lepisto, A.J. \& Hendricks, R.L. Immunity to latent viral infection: many skirmishes but few fatalities. Trends Immunol. 25, 230-234 (2004).

4. Kumar, A., Zhang, J. \& Yu, F.S. Toll-like receptor 3 agonist poly(l:C)induced antiviral response in human corneal epithelial cells. Immunology 117, 11-21 (2006)

5. Takeda, K., Kaisho, T. \& Akira, S. Toll-like receptors. Annu. Rev. Immunol. 21, 335-376 (2003).

6. Austin, B.A., James, C., Silverman, R.H. \& Carr, D.J. Critical role for the oligoadenylate synthetase/RNase L pathway in response to IFN-beta during acute ocular herpes simplex virus type 1 infection. J. Immunol. 175, 1100-1106 (2005).

7. Minkovitz, J.B. \& Pepose, J.S. Topical interferon alpha-2a treatment of herpes simplex keratitis resistant to multiple antiviral medications in an immunosuppressed patient. Cornea 14, 326-330 (1995). 
8. Staeheli, P., Haller, O., Boll, W., Lindenmann, J. \& Weissmann, C. Mx protein: constitutive expression in 3T3 cells transformed with cloned Mx cDNA confers selective resistance to influenza virus. Cell 44, 147-158 (1986).

9. Leib, D.A., Machalek, M.A., Williams, B.R., Silverman, R.H. \& Virgin, H.W. Specific phenotypic restoration of an attenuated virus by knockout of a host resistance gene. Proc. Natl Acad. Sci. USA 97, 6097-6101 (2000).

10. Jin, X., Qin, Q., Chen, W. \& Qu, J. Expression of toll-like receptors in the healthy and herpes simplex virus-infected cornea. Cornea 26, 847-852 (2007).

11. Hara, Y. et al. Alteration of TLR3 pathways by glucocorticoids may be responsible for immunosusceptibility of human corneal epithelial cells to viral infections. Mol. Vis. 15, 937-948 (2009).

12. Sarangi, P.P., Kim, B., Kurt-Jones, E. \& Rouse, B.T. Innate recognition network driving herpes simplex virus-induced corneal immunopathology: role of the toll pathway in early inflammatory events in stromal keratitis. J. Virol. 81, 11128-11138 (2007).

13. Liu, C.J., Wang, H. \& Lengyel, P. The interferon-inducible nucleolar p204 protein binds the ribosomal RNA-specific UBF1 transcription factor and inhibits ribosomal RNA transcription. EMBO J. 18, 2845-2854 (1999).

14. Unterholzner, L. et al. IFI16 is an innate immune sensor for intracellular DNA. Nat. Immunol. 11, 997-1004 (2010).

15. Hise, A.G. et al. Innate immune responses to endosymbiotic Wolbachia bacteria in Brugia malayi and Onchocerca volvulus are dependent on TLR2, TLR6, MyD88, and Mal, but not TLR4, TRIF, or TRAM. J. Immunol. 178, 1068-1076 (2007).

16. Tarabishy, A.B. et al. MyD88 regulation of Fusarium keratitis is dependent on TLR4 and IL-1R1 but not TLR2. J. Immunol. 181, 593-600 (2008).

17. Conrady, C.D., Thapa, M., Wuest, T. \& Carr, D.J. Loss of mandibular lymph node integrity is associated with an increase in sensitivity to HSV-1 infection in CD118-deficient mice. J. Immunol. 182, 3678-3687 (2009).

18. Conrady, C.D., Jones, H., Zheng, M. \& Carr, D.J.J. A functional type I interferon pathway drives resistance to cornea herpes simplex virus type 1 infection by recruitment of leukocytes. J. Biomed. Res. 25, 111-129 (2011).

19. Takaoka, A. et al. DAl (DLM-1/ZBP1) is a cytosolic DNA sensor and an activator of innate immune response. Nature 448, 501-505 (2007).

20. Jones, J.W. et al. Absent in melanoma 2 is required for innate immune recognition of Francisella tularensis. Proc. Natl Acad. Sci. USA 107, 9771-9776 (2010)

21. Chiu, Y.H., Macmillan, J.B. \& Chen, Z.J. RNA polymerase III detects cytosolic DNA and induces type I interferons through the RIG-I pathway. Cell 138, 576-591 (2009).

22. Sauer, J.D. et al. The N-ethyl-N-nitrosourea-induced Goldenticket mouse mutant reveals an essential function of Sting in the in vivo interferon response to Listeria monocytogenes and cyclic dinucleotides. Infect. Immunol. 79, 688-694 (2011).

23. Edelson, B.T. \& Unanue, E.R. Intracellular antibody neutralizes Listeria growth. Immunity 14, 503-512 (2001).

24. Cho, W.G. et al. Small interfering RNA-induced TLR3 activation inhibits blood and lymphatic vessel growth. Proc. Natl Acad. Sci. USA 106, 7137-7142 (2009).

25. Padovan, E., Spagnoli, G.C., Ferrantini, M. \& Heberer, M. IFN-alpha2a induces IP-10/CXCL10 and MIG/CXCL9 production in monocyte-derived dendritic cells and enhances their capacity to attract and stimulate CD8+ effector T cells. J. Leukoc. Biol. 71, 669-676 (2002).

26. Leib, D.A., Harrison, T.E., Laslo, K.M., Machalek, M.A., Moorman, N.J. \& Virgin, H.W. Interferons regulate the phenotype of wild-type and mutant herpes simplex viruses in vivo. J. Exp. Med. 189, 663-672 (1999).

27. Paludan, S.R., Bowie, A.G., Horan, K.A. \& Fitzgerald, K.A. Recognition of Herpes viruses by the innate immune system. Nat. Rev. Immunol. 11, 143-154 (2011).
28. Zhang, S.Y. et al. TLR3 deficiency in patients with herpes simplex encephalitis. Science 317, 1522-1527 (2007).

29. Guo, Y. et al. Herpes simplex virus encephalitis in a patient with complete TLR3 deficiency: TLR3 is otherwise redundant in protective immunity. J. Exp. Med. 208, 2083-2098 (2011).

30. Casrouge, A. et al. Herpes simplex virus encephalitis in human UNC-93B deficiency. Science 314, 308-312 (2006).

31. Zhang, Z., Yuan, B., Bao, M., Lu, N., Kim, T. \& Liu, Y.J. The helicase DDX41 senses intracellular DNA mediated by the adaptor STING in dendritic cells. Nat. Immunol. 12, 959-965 (2011).

32. Wei, W. et al. Expression of IFI 16 in epithelial cells and lymphoid tissues. Histochem. Cell Biol. 119, 45-54 (2003).

33. Xin, H., Curry, J., Johnstone, R.W., Nickoloff, B.J. \& Choubey, D. Role of IFI 16, a member of the interferon-inducible p200-protein family, in prostate epithelial cellular senescence. Oncogene 22, 4831-4840 (2003).

34. Ishikawa, H., Ma, Z. \& Barber, G.N. STING regulates intracellular DNAmediated, type I interferon-dependent innate immunity. Nature 461, 788-792 (2009).

35. Darnell, J.E. Jr, Kerr, I.M. \& Stark, G.R. Jak-STAT pathways and transcriptional activation in response to IFNs and other extracellular signaling proteins. Science 264, 1415-1421 (1994).

36. Medzhitov, R., Preston-Hurlburt, P. \& Janeway, C.A. Jr A human homologue of the Drosophila Toll protein signals activation of adaptive immunity. Nature 388, 394-397 (1997).

37. Conrady, C.D., Halford, W.P. \& Carr, D.J. Loss of the type I interferon pathway increases vulnerability of mice to genital herpes simplex virus 2 infection. J. Virol. 85, 1625-1633 (2011).

38. Sabroe, I., Parker, L.C., Dower, S.K. \& Whyte, M.K. The role of TLR activation in inflammation. J. Pathol. 214, 126-135 (2008).

39. Kurt-Jones, E.A. et al. Herpes simplex virus 1 interaction with Toll-like receptor 2 contributes to lethal encephalitis. Proc. Natl Acad. Sci. USA 101, 1315-1320 (2004).

40. Oshry, T. \& Lifshitz, T. Intravenous acyclovir treatment for extensive herpetic keratitis in a liver transplant patient. Int. Ophthalmol. 21, 265-268 (1997).

41. Al-Khatib, K., Williams, B.R., Silverman, R.H., Halford, W. \& Carr, D.J. Distinctive roles for $2^{\prime}, 5^{\prime}$-oligoadenylate synthetases and doublestranded RNA-dependent protein kinase $R$ in the in vivo antiviral effect of an adenoviral vector expressing murine IFN-beta. J. Immunol. 172, 56385647 (2004).

42. Adachi, O. et al. Targeted disruption of the MyD88 gene results in loss of IL-1- and IL-18-mediated function. Immunity 9, 143-150 (1998).

43. Garvey, T.L. et al. Inflammatory responses to pneumovirus infection in IFN-alpha beta R gene-deleted mice. J. Immunol. 175, 4735-4744 (2005).

44. Robertson, D.M. et al. Characterization of growth and differentiation in a telomerase-immortalized human corneal epithelial cell line. Invest. Ophthalmol. Vis. Sci. 46, 470-478 (2005).

45. Wuest, T.R. \& Carr, D.J. VEGF-A expression by HSV-1-infected cells drives corneal lymphangiogenesis. J. Exp. Med. 207, 101-115 (2010).

46. Takeshita, F., Suzuki, K., Sasaki, S., Ishii, N., Klinman, D.M. \& Ishii, K.J. Transcriptional regulation of the human TLR9 gene. J. Immunol. 173, 2552-2561 (2004).

47. Spurrell, J.C., Wiehler, S., Zaheer, R.S., Sanders, S.P. \& Proud, D. Human airway epithelial cells produce IP-10 (CXCL10) in vitro and in vivo upon rhinovirus infection. Am. J. Physiol. Lung Cell Mol. Physiol. 289, L85-L95 (2005).

48. Hoene, V., Peiser, M. \& Wanner, R. Human monocyte-derived dendritic cells express TLR9 and react directly to the CpG-A oligonucleotide D19. J. Leukoc. Biol. 80, 1328-1336 (2006). 